\title{
SSOPS based Solution to Improve QoS in VANET
}

\author{
Pallavi Sharma ${ }^{\mathrm{a}}$, Anil Sagar ${ }^{\mathrm{b}}$, Mohit Marwaha ${ }^{\mathrm{c}}$ \\ a Research Scholar, Beant College of Engineering and Technology, Gurdaspur, Punjab, India \\ apllvshrm145@gmail.com \\ ${ }^{b}$ Beant College of Engineering and Technology, Gurdaspur, Punjab, India \\ ${ }^{c}$ Beant College of Engineering and Technology, Gurdaspur, Punjab, India
}

Abstract: Vehicular Ad-hoc Networks (VANETs) is an emerging network technology derived from ad-hoc networks. This paper provides the state-of-the-art of VANETs and provides optimum proposal by improving Quality of Service (QoS.) Today, wireless systems are preferred over wired systems and these are gaining popularity as it provides wireless connectivity to the users irrespective of their geographic position, VANET is one of them. VANETs are installed to minimize the risk of road accidents and to improve passenger comfort by permitting the vehicles to exchange various types of data. In this paper, the Signal Strength based Optimum Path Selection (SSOPS) based solution on how to mitigate the QoS issues that exists while using the existing methods are discussed. Moreover, the solution has been tested using NS2 software using various parameters.

\section{Introduction}

Intelligent Transportation System (ITS) is an developing and varied subject with few of its essential parts overlapping or joining. One of its examples is: travel and transport information could be studied under Smart Cities agenda. Similarly, connected vehicles are an articulation of Machine-to-Machine (M2M) Communications and the Internet of Things (IoT). Therefore, Vehicular Ad-Hoc Network (VANET) networks is generally developed as a part of ITS.

ITS applications are in demand applications that without expressing intelligence aims to deliver advanced services connecting to various modes of traffic and transport management and enables various users to be better. It further informs and make safer, coordination alongside a smarter usage of transport networks.

MANET stands for "Mobile AdHoc Network". In such types of networks all the nodes within a network are mobile and these nodes can be deployed dynamically in random manner. Similarly, VANET stands for "Vehicular Adhoc Network" which is actually a subclass of MANET network class. However, the protocols, particularly, the routing protocols of both these types are entirely different from each other. One of the main types of MANET is VANET. From the advanced point of view, these two have quite similar nature. But few features are explicit for the VANETs that distinguishes it from the MANETs. Further, VANETs have unique characteristics in comparison to MANETs. Some of the main features of the VANETs includes: its heterogeneity, its mobility, topological constraints, density of vehicle, network frequently gets disconnected, topological changes, and many more.

There are some factors which must be considered while designing routing protocols for VANETs. 
These includes: mobility, security, and scalability of vehicles that communicates with each other. The objective of VANET design is to permit the links amid the vehicles/vehicles and fixed road side units that further leads to:

- V2V ad hoc Connection (Vehicle-tovehicle ad hoc networks)

It permits the communication directly without depending upon any support of fixed infrastructure. It provides high security, and safety.

- V2I network (Vehicle-to-Infrastructure networks)

It permits vehicles to connect with the road-side units specifically for data gathering purpose.

- Hybrid architecture

It is actually a combination of V2I and V2V. In such cases, a a multi-hop/single hop fashion can be used by vehicles for communicating with the roadside infrastructure. It further depends on the distance. It also allows larger distances linkage to the vehicle/Internet that are at distant places.

\section{Related Work}

[1] presented a novel approach to the management of networks adhoc in nature. In this paper, the authors found the amendments to the BellmanFord routing algorithm as per the protocol (RIP). This is done to make it more suitable for dynamic $\&$ self-starting network mechanism.

[2] described a complex source routing protocol for routing in ad hoc networks. The protocol in this case adjusts rapidly to the routing changes if host movements are regular, however, it requires little or no overhead if there are frequently less host movements. less frequently. Finally. The authors concluded that the protocol performs well under the conditions of host density and movement speeds.
[3] presented fisheye state routing, a novel routing protocol for wireless ad hoc networks (FSR). To minimize routing upgrade overhead in large networks, FSR introduces the concept of multilevel fisheye scope. The frequency with which nodes exchange connection state entries with their neighbors is determined by the distance to the destination. Nodes create the topology map of the entire network and compute optimal routes using connection state entries. In a mobile, ad hoc environment, simulation experiments show that FSR is an easy, reliable, and scalable routing solution.

[4] based on research into previous work in the field, identified the functions of a unidirectional IP-based file delivery protocol. The authors study several historical works on file distribution protocols to provide context for the current state of the art, FLUTE, which is being implemented as a standard in the Internet engineering task force. They also compare various approaches and introduce items for future research in this field. [5] VANET's aim is to provide adequate quality of service (QoS) for real-time safety applications while also supporting commercial services. This paper proposes a multi-channel wireless networking architecture and protocol for scenarios in which roadside infrastructure provides commercial services. The IEEE 802.11 wireless LAN protocol is extended in this approach to allow for the scheduling of periodic safety messages in a "safety channel." It facilitates nontime-critical communications in different, nonsafety "service networks" at the same time. This arrangement also maximizes service channel access time while preserving the necessary QoS for safety applications, according to the results. The paper ends with simulations that affirm the architecture's and protocol's appealing properties. [6] said that the scenario's most challenging challenge is dealing with regular path breaks caused by the complex mobility of vehicles on the 
track. The dynamic mobility of vehicles on highways can be used to estimate how long a path between a vehicle that requires Internet access and the gateway that provides access to the Internet would take. The number of path failures can be greatly reduced if route lifetimes can be accurately predicted. They simulate the protocol's success and find that it reduces route failures significantly as compared to protocols that don't use preemptive routing. Furthermore, they discover that the overhead of preemptive routing is held to a minimum thanks to PBR's ability to predict routes. [7] argued that in recent years, there has been research on active safety systems with an emphasis on environmental sensing. As a result, vehicles are fitted with sensors that record data about the current traffic situation and potential hazards in the region. Additional information outside of the range of the sensors' region is needed for anticipatory driving. This can be accomplished by integrating data transfer between vehicles, which allows for the collection of data from a wider region and thereby increases the system's anticipatory capabilities. The organization of access to the medium is an important feature of data sharing between vehicles. It must be capable of dealing with all of the issues that arise in mobile ad-hoc networks. In vehicular ad-hoc networks, the secret station issue is particularly problematic (VANETs). However, due to the high density of nodes, high dynamics, and low data rate, a reasonable and efficient access to the medium is needed. They present a medium access scheme for vehicular ad-hoc networks that is focused on vehicle clustering in this paper. As a result, the impact of secret stations can be reduced, resulting in more efficient data transfer than IEEE 802.11-based systems.

[8] DDoS is a spy-on-spy game between attackers and detectors, according to him. Attackers are imitating network traffic patterns in order to fool detection algorithms that depend on these characteristics. Distinguishing mimicking DDoS attacks from huge legitimate network access is an open challenge. They discovered that zombies use managed function(s) to pump attack packages to the victim, so attack flows to the victim often share certain properties, such as package delivery behaviours, that legitimate flows do not have in a short time span. Based on this observation, when suspicious flows to a server appear, they begin to measure the distance between suspicious flows in terms of package delivery activity. It's a DDoS attack if the distance is less than a certain threshold; otherwise, it's a legal access. Their research and preliminary tests show that the proposed approach can effectively and efficiently distinguish mimicking flooding attacks from legitimate accessing.

[9] introduced proposed a model named "channel busy time" for evaluating the solution space of a vehicular beaconing system. The main aim for designing this model is to transfer information both vital and sufficient for co-operative adaptive cruise control \& VANETs. They also identified that the solution space is 3-dimentional in nature and is based on the number of vehicles.

[10] presented an adaptive decentralized method for information dissemination in VANETs with an aim to prove the optimum performance of adaptive approaches over statistical methods.

[11] said that VANET is a subset of mobile ad hoc networks that offers a unique approach to Intelligent Transportation Systems, according to the report (ITS). For smart ITS, a survey of routing protocols in VANET is essential and appropriate. The author addressed the benefits and drawbacks of different routing protocols for vehicular ad hoc networks, as well as their implementations. It investigates the impetus for the design as well as the progression of these routing protocols. Finally, the paper ends with a table that compares the different VANET routing protocols. 
[12] An Eta squared is a statistic that calculates the proportion of a dependent variable's total variance that is linked to membership in different groups identified by an independent variable.

The effects of other independent variables and interactions are partialled out in partial eta squared, which is a similar measure. The evolution of these steps is discussed, as well as their characteristics. The two measures have been mixed up in the research literature in the past, partly due to a labelling error in the output produced by some versions of the statistical package SPSS.

In today's educational research literature, partial eta squared is overwhelmingly used as an indicator of effect size. Although there are good reasons for this, it is important to interpret all steps with caution. A list of the main characteristics of eta squared and partial eta squared is presented at the end of the paper.

[13] presented different IoT projects that are in progress that guarantee to close the hole among poor and rich, improve conveyance of the world's assets to the individuals who need them most, and assist us with understanding our planet so we can be more proactive and less receptive. All things being equal, a few obstructions exist that threaten to moderate IoT improvement, including the change to IPv6, having a typical arrangement of guidelines, and creating fuel hotspots for millions - even billions - of moment sensors. Nonetheless, as organizations, governments, norms bodies, and the scholarly world work together to tackle these difficulties, IoT will keep on advancing. The objective of this paper, consequently, is to make individuals acquainted with IoT and comprehend its latent capacity.

[14] presented late headways in wireless advancements that brought about the development of VANETs. In such organizations, the restricted inclusion of $\mathrm{Wi}-\mathrm{Fi}$ and the high portability of the hubs produce successive geography changes and organization discontinuities. Consequently, and considering that there is no focal director substance, steering bundles through the organization is a difficult assignment. Thusly, offering a productive steering system is pivotal to the arrangement of VANETs. This paper manages the ideal boundary setting of the optimized link state routing (OLSR), which is a MANET routing protocol, by characterizing an enhancement issue. Along these lines, a progression of agent metaheuristic calculations (particle swarm optimization, differential development, hereditary calculation, and reproduced toughening) is concentrated in this paper to discover naturally ideal arrangements of this issue of optimization. Likewise, a bunch of sensible VANET situations have been characterized to precisely assess the exhibition of the organization under our programmed OLSR. In the examinations, our tuned OLSR setups bring about better nature of administration $(\mathrm{QoS})$ than the standard solicitation for remarks (RFC 3626), just as a few human specialists, making it agreeable for usage in VANET designs.

[15] focused exactly on the design/architecture of efficient broadcast-based content delivery system with a motive to minimize the content delivery time to as minimum as possible. For this, they firstly optimized the performance by seeking the optimal packet size \& then they developed a full architecture to achieve efficient content distribution.

[16] talked about an OpenStreetMap that allows internet workers to edit \& contribute geospatial data. The collected data through OpenStreetMap is then complemented with digitized open-source aerial photography and free maps from the governmental and commercial sources.

[17] proposed a probabilistic trust mindful Data Replication methodology for online video webbased applications. The proposed strategy's 
outcome suggests that with higher LSVs and low versatility, the replica cost diminishes.

[18] presented a novel Secure WLAN Authentication Scheme (SWAS) to enhance the security by protecting the messages using cryptographic techniques. Further, this scheme minimizes the network overhead \& communication cost.

[19] gave a taxonomy on clustering in VANETs along with a comprehensive study of all the existing techniques. The study provided for various proposals permits various users to select one of the proposals with respect to its merits.

[20] proposed Bayesian Coalition Game (BCG) in order to overcome the issue of substance dispersion to vehicles from the point of view of learning automata \& BCG. Further, the proposed plot is found to be most powerful method for IoV climate.

\section{Problem Statement}

The environment in which vehicular networks operate is extremely dynamic, and includes extreme configurations. In highways, a relative speed of $300 \mathrm{~km} / \mathrm{h}$ may occur, while density of nodes may be 1-2 vehicles per kilometre in less busy roads. Because of the relative movement of the vehicles, the connectivity among nodes could last only few seconds, and fail in unpredictable ways. Hence our research aims to increase QoS in vehicular area network by increasing packet delivery ratio, Throughput, and decreasing delay in a network.

\section{Results and Discussions}

After going through the detailed brief literature survey, it is noticed that because of the high mobility of vehicles, it will lead to various challenges in order to maintain communication links between the vehicles in a network. Due to this, the packet drop rate is very high in a network, which will further lead to low QoS. In order to mitigate this problem signal strength and congestion aware mechanism to improve QoS for VANET has been proposed. The parameters used to achieve the optimum solution are shown in table 1

A detailed simulation model based on NS2 is used. Figure 1 shows the animation for the proposed approach.

Table 1 Simulation Parameters

\begin{tabular}{|c|c|}
\hline $\begin{array}{c}\text { SIMULATION } \\
\text { PARAMETERS }\end{array}$ & VALUES \\
\hline Area & $2528 * 2068 \mathrm{~m}$ \\
\hline Number of Nodes & 100 \\
\hline Queue Length & 50 \\
\hline Protocol & AODV \\
\hline Scenario & Random \\
\hline Queue Type & Drop Tail \\
\hline MAC Layer protocol & 802.11 \\
\hline Data Rate & 4 Packets/Sec \\
\hline Number of connections & Varied (5, 10, 15, 20, \\
\hline
\end{tabular}

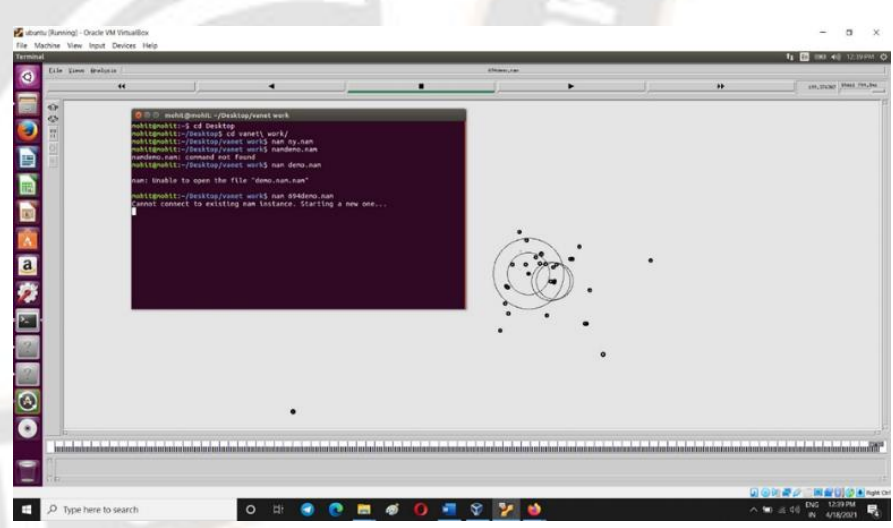

Figure 1 Nam command to run Animation in NS2

\section{Performance Metrics}

\section{Packet Delivery ratio}

The packet delivery ratio in this simulation is defined as the ratio between the number of packets sent by the constant bit rate sources (CBR, application level) and the number of receiver 
packets per CBR receiver in the destination table. The values achieved after implementing the proposed and existing solution are shown in table 2 and figure 2 below:

Table 2: Packet delivery ratio

\begin{tabular}{|c|c|c|}
\hline $\begin{array}{c}\text { Number of } \\
\text { Mobile } \\
\text { Connections }\end{array}$ & $\begin{array}{c}\text { Existing } \\
\text { Technique } \\
\text { (Kalman Filter } \\
\text { Technique) }\end{array}$ & $\begin{array}{c}\text { Proposed } \\
\text { Technique } \\
\text { (SSOPS) }\end{array}$ \\
\hline 5 & 0.702 & 0.7193 \\
\hline 10 & 0.6968 & 0.7266 \\
\hline 15 & 0.6433 & 0.6986 \\
\hline 20 & 0.6492 & 0.6914 \\
\hline 25 & 0.6376 & 0.6483 \\
\hline
\end{tabular}

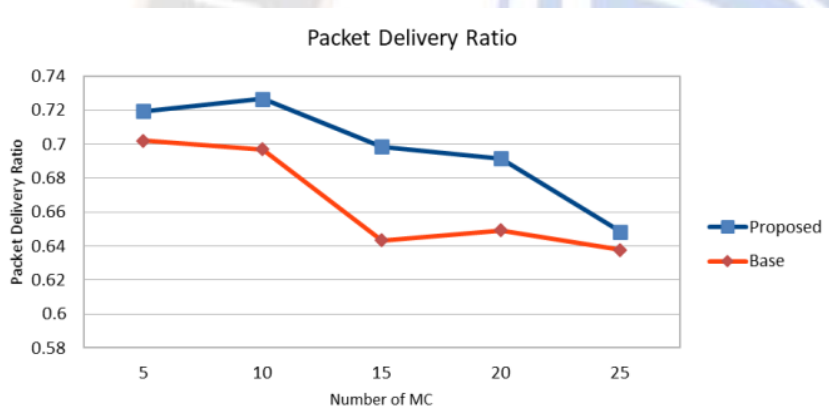

Figure 2. Packet Delivery Ratio

PDR for the proposed work is better at every point of calculation. When the number of mobile connections is 5 , then the value achieved for the proposed work is far good in comparison to the base work that is for the proposed work, PDR is 0.7193 and for base, the value is 0.702 . Moving further, when the number of connections is increased to 10, the value for the proposed work is again more efficient, that is 0.7266 , whereas 0.6968 is the value achieved for base work scenario. This trend, is similar for the number of mobile connections 15,20 , and 25 that is values are higher and better for proposed work in comparison to the base work.
2. End-to-end delay: This metric represents an average end-to-end delay and indicates how long it takes for a packet to travel from the source to the target application level. It includes all the possible delays caused by buffering during the latency of the route detection, transmission delays in the MAC, the queue in the interface queue and the propagation and transfer time. It is measured in seconds.

When the number of mobile connections is set at 15 and 25 number of mobile connections, the delay value is maximum for the proposed work, while it is less for base work. Further, when at varying number of mobile connections, the proposed work experiences least delay in comparison to base work. Refer table 3 and figure 3 for the values obtained after implementing both the existing and proposed solution in NS2.

Table 3: Average End-to-End Delay

\begin{tabular}{|c|c|c|}
\hline $\begin{array}{c}\text { Number of } \\
\text { Mobile } \\
\text { Connections }\end{array}$ & $\begin{array}{c}\text { Existing } \\
\text { Technique } \\
\text { (Kalman } \\
\text { Filter } \\
\text { Technique) }\end{array}$ & $\begin{array}{c}\text { Proposed } \\
\text { Technique } \\
\text { (SSOPS) }\end{array}$ \\
\hline 5 & 575.911 & 417.447 \\
\hline 10 & 483.365 & 335.968 \\
\hline 15 & 400.652 & 460.565 \\
\hline 20 & 479.72 & 459.539 \\
\hline 25 & 705.827 & 720.679 \\
\hline
\end{tabular}




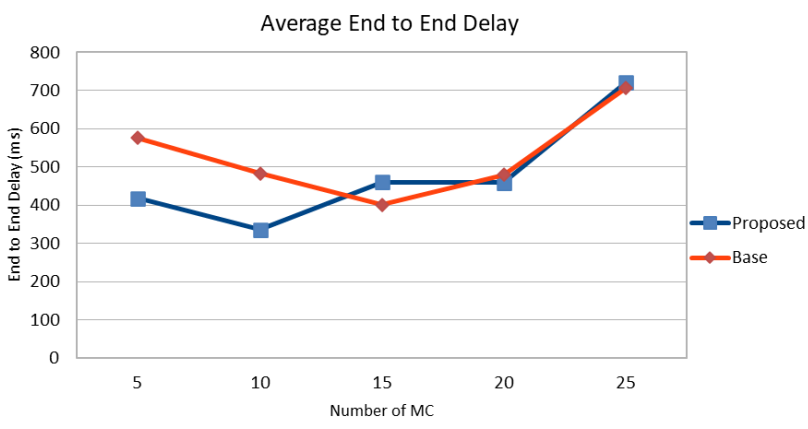

Figure 3 Average End to End Delay

\section{Conclusion}

In this thesis, we have proposed an optimum solution for VANET by reducing the error probability in it. The existing approach measured the distance via average speed change of a vehicle, which leads to high error rates specially whenever there is a road diversion or path change. The existing technique used Kalman technique/method to measure the distance. However, the disadvantage of this approach is that it leads to high error probability. Thus, the same is mitigated using SSOPS (proposed technique) which is an improvement over the Kalman technique.

\section{References}

[1] C. E. Perkins and P. Bhagwat, "Highly dynamic destination-sequenced distance-vector routing (DSDV) for mobile computers," in ACM SIGCOMM computer communication review, vol. 24, no. 4. ACM, London, UK, Aug 31 - Sep 2, 1994, pp.234244.

[2] D. B. Johnson and D. A. Maltz, "Dynamic source routing in ad hoc wireless networks," in Mobile computing. Springer, 1996, pp. 153-181.

[3] G. Pei, M. Gerla, and T.-W. Chen, "Fisheye state routing: A routing scheme forad hoc wireless networks," in IEEE International Conference on Communications, ICC 2000, vol. 1. IEEE, New Orleans, LA, 18-22 June, 2000, pp. 70-74.

[4] T. Paila, "Unidirectional ip-based mass file delivery protocol," in 1st International symposium on Wireless Communication Systems, 2004. IEEE, Mauritius, 20-22Sep, 2004, pp. 235-239.

[5] T. K. Mak, K. P. Laberteaux, and R. Sengupta, "A multi-channel vanet provid-ing concurrent safety and commercial services," in Proceedings of the 2nd ACM international workshop on Vehicular ad hoc networks. ACM, Cologne, Germany, September 02, 2005, pp. 1-9.

[6] V. Namboodiri and L. Gao, "Prediction-based routing for vehicular ad hoc net-works," IEEE Transactions on Vehicular Technology, vol. 56, no. 4, pp. 2332-2345,2007.

[7] Y. G unter, B. Wiegel, and H. P. Grossmann, "Cluster-based medium access scheme for vanets," in IEEE Intelligent Transportation Systems Conference, ITSC 2007.IEEE, 2007, pp. 343-348.

[8] S. Yu, W. Zhou, and R. Doss, "Information theorybased detection against network behavior mimicking ddos attacks," IEEE Communications Letters, vol. 12, no. 4, pp. 318-321, 2008.

[9] M. Van Eenennaam, W. K. Wolterink, G. Karagiannis, and G. Heijenk, "Exploring the solution space of beaconing in VANETs," in IEEE Vehicular Networking Conference (VNC), 2009. IEEE, Tokyo, Japan, 28-30 Oct, 2009, pp. 1-8.

[10] M. Bakhouya, J. Gaber, and P. Lorenz, "An adaptive approach for information dissemination in vehicular ad hoc networks," Journal of Network and Computer Applications, vol. 34, no. 6, pp. 19711978, 2011.

[11] R. Kumar and M. Dave, "A comparative study of various routing protocols in VANET," arXiv preprint arXiv:1108.2094, 2011.

[12] J.T. Richardson, "Eta squared and partial eta squared as measures of effect size in educational research," Rev. Educ. Res. 6(2), 135-147, 2011.

[13] D. Evans, "The internet of things: How the next evolution of the internet is changing everything," CISCO white paper, vol. 1, pp. 1-11, 2011.

[14] J. Toutouh, J. Garcia-Nieto, and E. Alba, "Intelligent OLSR routing protocol optimization for VANETs," IEEE Transactions on Vehicular Technology, vol. 61, no. 4, pp. 1884-1894, 2012.

[15] C. T. Calafate, G. Fortino, S. Fritsch, J. Monteiro, J.-C. Cano, and P. Manzoni, "An efficient and robust content delivery solution for ieee $802.11 \mathrm{p}$ vehicular environments," Journal of Network and Computer Applications, vol. 35, no. 2, pp.753-762, 2012.

[16] K. Curran, G. Fisher, J. Crumlish, Openstreetmap, Int. J. Interact. Commun. Syst. Technol. 2, 69-78, 2012.

[17] N. Kumar and J. Kim, "Probabilistic trust aware data replica placement strategy for online video streaming applications in vehicular delay tolerant networks," Mathematical and Computer Modelling, vol. 58, no. 1, pp. 3-14, 2013. 
[18] R. Singh and T. P. Sharma, "A secure wlan authentication scheme," IEIE Transactions on Smart Processing \& Computing, vol. 2, no. 3, pp. 176-187, 2013.

[19] R. S. Bali, N. Kumar, and J. J. Rodrigues, "Clustering in vehicular ad hoc networks: taxonomy, challenges and solutions," Vehicular communications, vol. 1, no. 3, pp.134-152, 2014.

[20] N. Kumar, J. J. Rodrigues, and N. Chilamkurti, "Bayesian coalition game as-a-service for content distribution in internet of vehicles," IEEE Internet of Things Journal, vol. 1, no. 6, pp. 544-555, 2014.

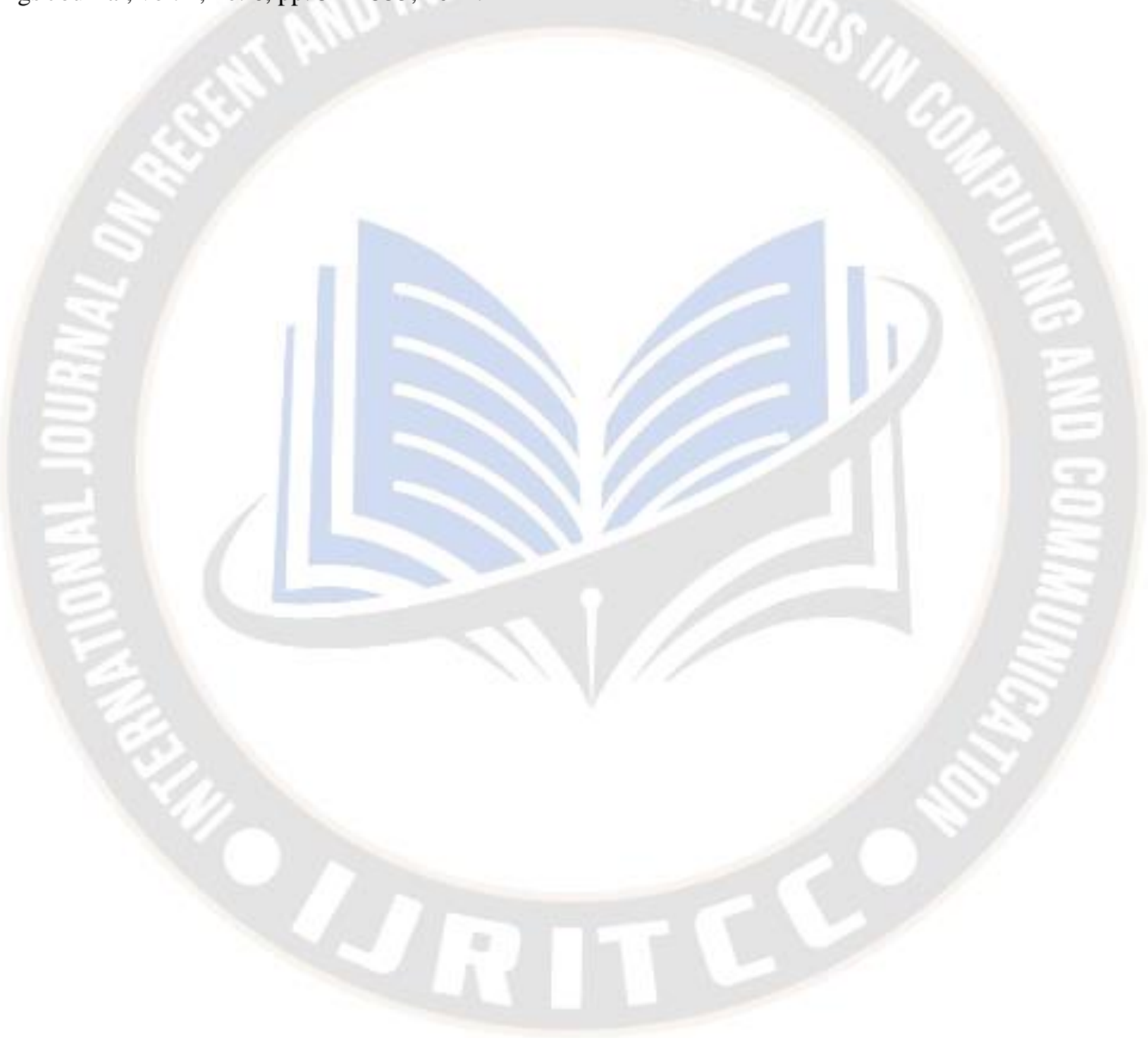

